\title{
Comparative Human Landing Catch and CDC Light Trap in Mosquito Sampling in Knowlesi Malaria Endemic Areas in Peninsula Malaysia
}

\author{
Ahmad Rohani' ${ }^{1}$, Abd Rahman Aidil Azahary ${ }^{1,2}$, Mohamed Nor Zurainee², \\ Wan Mohd Ali Wan Najdah', Ismail Zamree', Mohd Omar Hanif ${ }^{1}$, Mohd Majid Ariffin1, \\ Hamid Zuhaizam1, Ismail Suzilah ${ }^{3}$, Han Lim Lee ${ }^{1}$ \\ ${ }^{1}$ Medical Entomology Unit, Institute for Medical Research, Kuala Lumpur, Malaysia \\ ${ }^{2}$ Department of Parasitology, University of Malaya, Kuala Lumpur, Malaysia \\ ${ }^{3}$ School of Quantitative Sciences (SQS), UUM College of Arts and Sciences, University Utara Malaysia, Sintok, \\ Malaysia \\ Email:rohania@imr.gov.my, aidilbiosc@gmail.com,w_najdah@yahoo.com,kumbanglaut@yahoo.com, \\ hanifakifadila@yahoo.com, ariffin@imr.gov.my,zuhaizam@imr.gov.my, \\ zuraineemn@um.edu.my,halizus@uum.edu.my
}

Received 20 October 2015; accepted 27 November 2015; published 30 November 2015

Copyright $(\underset{2016}{ } 2 \mathrm{by}$ authors and Scientific Research Publishing Inc.

This work is licensed under the Creative Commons Attribution International License (CC BY). http://creativecommons.org/licenses/by/4.0/

(c) (;) 0 pen Access

\begin{abstract}
Adult mosquito sampling techniques are essential for monitoring transmission of malaria and other mosquito borne infections. Preference for any sampling technique depends on both its field efficiency and the characteristics of local vector populations. Surveys on adult mosquitoes using Human Landing Catch (HLC) and $\mathrm{CO}_{2}$-baited CDC light trap (CDC-LT) techniques were conducted in several knowlesi malaria endemic areas between the months of March to December 2012 in several states of Peninsula Malaysia. These two techniques were relatively compared to determine the preferences of anopheline mosquitoes towards $\mathrm{CO}_{2}$-baited CDC-LT technique using HLC technique as the reference method. Cx. gelidus, An. maculates and An. introlatus were the main three species collected by HLC technique, whereas the species collected by $\mathrm{CO}_{2}$-baited $\mathrm{CDC}$-LT technique were mostly An. cracens, $\mathrm{Ar}$. durhami and Coquillettidia species. Most of the Anopheles species were collected almost exclusively by the human collectors except for An. cracens and An. introlatus which were collected using both techniques. Anopheles cracens was the most dominant species captured using $\mathrm{CO}_{2}$-baited $\mathrm{CDC}$-LT technique. This is the first report showing An. cracens was caught using $\mathrm{CO}_{2}$-baited CDC-LT technique in Malaysia.
\end{abstract}

Keywords

Anopheles cracens, Human Landing Catch, CDC Light Trap

How to cite this paper: Rohani, A., et al. (2016) Comparative Human Landing Catch and CDC Light Trap in Mosquito Sampling in Knowlesi Malaria Endemic Areas in Peninsula Malaysia. Advances in Entomology, 4, 1-10. 


\section{Introduction}

The burden of malaria in many parts of Malaysia has decreased substantially due to malaria control activities implemented by Ministry of Health Malaysia since 1960's. However, a fifth species, Plasmodium knowlesi that was originally described as malaria parasite of the long-tailed macaque monkeys was being reported from time to time after the first case of knowlesi malaria was reported in humans in 1965 [1]. Subsequent extensive vector studies in the country showed that knowlesi malaria might not be a serious problem since the vectors were found only in the deep jungle. However, the situation has now changed. It is apparent now that deforestation in many areas has brought the monkeys and the forest dwelling Anopheles vectors closer to human habitation. These Anopheles mosquitoes and the nonhuman primates now colonize villages and plantations. With the reduction in the number of malaria cases, the population would have lost their immunity due to reduced exposure. This coupled with the presence of vectors and the infected long-tailed macaques is contributing to the increase in the number of simian malaria cases among humans over the years.

Plasmodium knowlesi has more recently been recognized as an important zoonotic malaria species in Malaysia [2] and cases have been reported throughout the country. The cases are on the increase year after year anddeath due to knowlesi malaria has also been reported [3]. Since 2013, P. knowlesi infection recorded the highest number of cases with $46.4 \%$ of all the malaria cases followed by $P$. vivax, $27.4 \%$ and $P$. falciparum, $16.7 \%$ respectively [4]. Furthermore, most of the knowlesi malariacases are occurring in malaria free areas where some vector species like An. latens and An. cracens are found [5].

In order to measure malaria transmission, a good knowledge about its vectors is required. Furthermore, assessing the success of malaria vector control requires a robust and accurate entomological monitoring system. Adult mosquito sampling techniques are essential for monitoring transmission of malaria. However, preference for any sampling technique depends on both its field efficacy and the characteristics of vector populations. $\mathrm{Hu}-$ man biting rate, an important parameter for assessing malaria transmission and evaluating vector control intervention, is commonly estimated by human landing catch (HLC) [6]. This technique although is the most reliable means of characterizing mosquito biting density on humans; it is logistically demanding and can present some ethical concerns by exposing people to mosquitoes that might be infected with malaria. However, this risk can be eliminated by providing all mosquito catchers with prophylaxis while participating in mosquito sampling. The logistically demanding nature of this works means there would be of great value in finding a similar sensitive but human exposure-free alternative technique.

A trapping device is the most common and the simplest tool used for the collection of mosquitoes, mainly for its surveillance, vector relative density and abundance and its control. Although various studies have been carried out using different trapping techniques, collection was not so successful for each type of trap has its own limitations. Various modifications have been added to increase the efficiency of the trap. The Centres for Disease Control light trap (CDC-LT) technique, have gained widespread application especially due to their reliability, robustness, ease of use, lower costs of application as compared to HLC technique. In order to increase its effectiveness, modification such as the use of $\mathrm{CO}_{2}$ or dry ice as attractant is applied [7]. The established role of $\mathrm{CO}_{2}$ as a mosquito attractant made it feasible to use as a standard in the sampling of mosquito population. $\mathrm{CO}_{2}$ elicited two distinct actions: firstly, it acted as an independent attractant and secondly, it interacted with warm moist convection currents limited by the host at close range together with other odour factors a distance from host. It has been reported that light trap technique supplemented with $\mathrm{CO}_{2}$ showed synergistic effect toward various species of mosquitoes [8] [9].

Various entomological indicators and sampling techniques are used to monitor and evaluate the impact of any vector control interventions. Therefore, in any particular investigation, there should be a careful selection of the most appropriate technique so that the most meaningful and pertinent information is collected. In view of the scarcity of trained manpower, high cost of operation and the need for testing of sampling technique capable of alleviating or replacing the routine mosquito collection technique in malaria control program in Peninsula Malaysia, it was considered important to investigate the efficiency of CDC-LT and HLC techniques for the sampling of malaria vectors. This study was part of a vector ecology/arthropod-borne pathogen research program designed to evaluate the threat that mosquito-borne pathogen pose to human and animals in knowlesi malaria endemic areas. The present study compared mosquito catches using $\mathrm{CO}_{2}$-baited CDC-LT technique against HLC technique in five knowlesi malaria endemic areas in Peninsula Malaysia. 


\section{Materials and Methods}

\subsection{Study Site}

This study was carried out in the following selected areas: Kg. Mela (Kuala Lipis, Pahang) [4 $4^{\circ} 3^{\prime} 48.42^{\prime \prime N}$ $\left.102^{\circ} 00^{\prime} 33.53 " \mathrm{E}\right]$, Lata Cemerung (Dungun, Terengganu) [4 $4^{\circ} 45^{\prime} 21.01^{\prime \prime N} 102^{\circ} 51^{\prime} 08.79 " \mathrm{E}$ ], Pos Dipang (Kampar, Perak) [4 $\left.4^{\circ} 22^{\prime} 10.38^{\prime \prime N} 101^{\circ} 16^{\prime} 2.64 " E\right]$, Mersing (Johor) [2 $\left.2^{\circ} 41^{\prime} 24.18^{\prime \prime N} 103^{\circ} 48^{\prime 2} 24.189 " E\right]$ and Kg. Sokor (Tanah Merah, Kelantan) [5 $47^{\prime} 38.67^{\prime \prime N ~} 101^{\circ} 48^{\prime} 22.54^{\prime E}$ ]. The area selection was based on malaria cases reported for the year 2011 and 2012. The five villages are situated in a knowlesi transmission risk areas (Figure 1). More than $80 \%$ of the villagers exploited their land for rubber and oil palm plantations to a lesser extent for fruit orchards.

\subsection{Mosquito Collection}

Mosquito sampling was conducted within the fruit orchard, oil palm and rubber tree plantations, about $1-2 \mathrm{~km}$ from the nearest house. Collections were performed from March to December 2012 based on Completely Randomized Design. The factor was technique used to catch the mosquitoes which has two factor levels (HLC and $\mathrm{CO}_{2}$-baitedCDC-LT techniques) and the response variable was number of mosquitoes caught. The study was conducted separately for the five areas due to limited resources such as man power and materials.

Mosquitoes were collected for three consecutive nights for each area. The samplings were conducted outdoors from 1800 to $2400 \mathrm{~h}$. Both techniques were performed on the same night.HLC technique consists of three teams of two catchers per team where the six catchers have similar ability and skills in conducting the study. They were randomly assigned according to the three teams and the teams were also randomly assigned for different location in the same study area. All mosquitoes that landed on the bare legs were caught using $50 \times 19 \mathrm{~mm}$ glass vials which were subsequently plugged with cotton wool. During collections, all catchers were equipped with moderate intensity torch lights to ensure that only mosquitoes were caught.

A total of nine $\mathrm{CO}_{2}$-baitedCDC-LT technique were fixed randomly at each area. The $\mathrm{CO}_{2}$-baitedCDC-LT technique is operated by four $1.5 \mathrm{~V}$ batteries which drive the suction fan and a $1.5 \mathrm{w}$ bulb. A collapsible collecting bag fitted in a metal frame hangs below the trap. The $\mathrm{CO}_{2}$ is emitted from $0.5 \mathrm{~kg}$ dry ice placed in a six

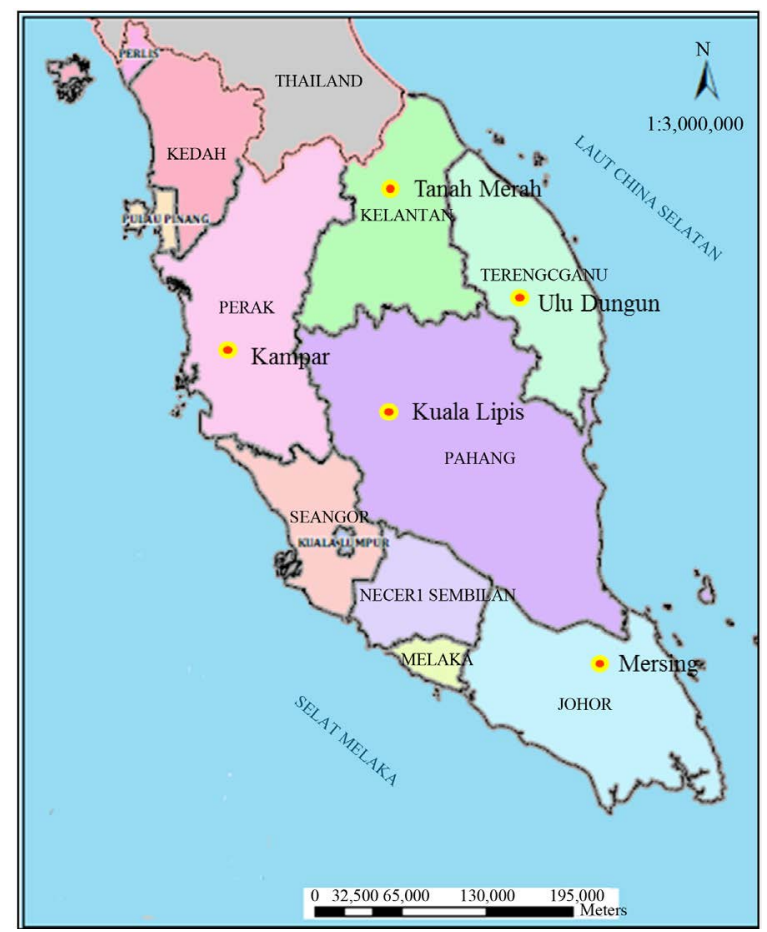

Figure 1. Map of Malaysia showing the five study localities where adult mosquitoes were collected. 
square inches ice box suspended adjacent and slightly above the light trap. The $\mathrm{CO}_{2}$ gas was supplied to the trap through a rubber tube connecting the ice box and the trap. The point of emission was above the suction fan and below the cover. All female mosquitoes from each trap were combined and the number of mosquitoes was calculated.

All captured mosquitoes were identified microscopically in the field laboratory using standard taxonomic keys [1] [10] the following morning and segregated according to species, village and date.

\subsection{Statistical Analysis}

Although other insects were present, only female mosquitoes collected during the study were counted and identified to species. Two Proportion Z-Tests and Fisher Exact Tests were conducted in determining the significant difference between mosquitoes captured using $\mathrm{HLC}$ and $\mathrm{CO}_{2}$-baited CDC-LT techniques for each of the five areas. All data were analyzed using statistical software SPSS 19.

\subsection{DNA Extraction and PCR}

DNA was extracted from An. cracens mosquitoes using the DN easy tissue kit (Qiagen, Germany), according to manufacturer's protocol. The extracted DNA was kept at $-20^{\circ} \mathrm{C}$ until required.

\subsection{Molecular Identification and Sequencing Targeting the Internal Transcribed Spacer 2 (ITS2)}

To confirm the species of An. cracens, PCR was carried on targeted atITS2 gene. The ITS2 was amplified using primers ITS2A (5' TGTGAACTGCAGGACA 3') and ITS2B (5' TATGCTTAAATTCAGGGGGT 3') [11], [12]. Each reaction mixture of $50 \mu \mathrm{L}$ contained $5 \mu \mathrm{L}$ of mosquito DNA template, primers ITS2A and ITS2B, 0.2 $\mu \mathrm{M}$ respectively, and $25 \mu \mathrm{l} 2 \times$ MyTaq Mix (Bioline, USA). PCR reactions were performed using Mastercycler Thermal Cycler (Eppendorf, Germany). The PCR conditions were as follows: 1) denaturation at $94^{\circ} \mathrm{C}$ for 5 minutes, 2) 35 cycles of amplification at $94^{\circ} \mathrm{C}$ for 1 minute, annealing step at $51^{\circ} \mathrm{C}$ for 1 minute with elongation step at $72^{\circ} \mathrm{C}$ for 2 minutes, followed by 3) final elongation step of 10 minutes at $72^{\circ} \mathrm{C}$ and a hold temperature of $4^{\circ} \mathrm{C}$. Amplicons were subjected to electrophoresis on $1.5 \%$ agarose gels (Promega, Madison, WI).

\subsection{Ethical Approval}

Free and informed consent was obtained from collectors performing HLC technique. Community consent had been obtained beforehand in all selected villages. This study was approved by Medical Research \& Ethics Committee, ministry of Health, Malaysia (NMRR-13-267-15323).

\section{Results}

A total of 1416 mosquitoes were collected during the study. Identification based on microscopy examination showed that they representing six genera and 34 species. A summary of mosquito collection by HLC and $\mathrm{CO}_{2}$-baitedCDC-LT techniques from the five study areas are presented in Table 1 . The number of mosquito indicated for each area was the total from the three samplings performed at individual area. Collections for different area however were done at different time due to limitation such as logistics and man power. The dominant species varied in each study area, but it was clear that An. cracens and $C x$. gelidus were present in a significant numbers in all study areas. In general, An. cracens was the most abundant species (14.9\%) collected followed by Cx. gelidus (14.3\%), An. introlatus (9.6\%), An. maculatus (9.5\%), and Ar. durhami (5.4\%). The highest number of An. cracens was obtained from Kampong Mela (Kuala Lipis Pahang) $(n=92)$ followed by Lata Cemerong (Dungun Terengganu) $(\mathrm{n}=54)$. An. introlatus and An. maculates were the predominant species for Mersing (Johor) (43.6\%) and Kg. Sokor (Tanah Merah Kelantan) (25.6\%) respectively. Other species were present in smaller percentages.

HLC and $\mathrm{CO}_{2}$-baited CDC-LT techniques located at five knowlesi endemic areas for 15 trap nights were compared to determine the technique efficiency based on number of mosquitoes captured by both techniques. Out of 34 species captured only 10 species namely Ae. albopictus, Ae. caecus, Ae. niveus, Ar. durhami, Coquillettidia spp., Cx. quinquefasciatus, Ma. bonnae, An. cracens, An. introlatus and An. letifer were captured by 
Table 1. The number of mosquitoes collected from all study areas.

\begin{tabular}{|c|c|c|c|c|c|c|c|c|c|c|c|}
\hline \multirow[b]{2}{*}{ Mosquito species } & \multicolumn{2}{|c|}{ Kg. Mela, Pahang } & \multicolumn{2}{|c|}{$\begin{array}{l}\text { Cemerong, } \\
\text { Terengganu }\end{array}$} & \multicolumn{2}{|c|}{ Pos Dipang, Perak } & \multicolumn{2}{|c|}{$\begin{array}{l}\text { Kg Sokor, } \\
\text { Kelantan }\end{array}$} & \multicolumn{2}{|c|}{ Mersing, Johor } & \multirow[b]{2}{*}{ Total } \\
\hline & HLC & CDC-LT & HLC & CDC-LT & HLC & CDC-LT & HLC & CDC-LT & HLC & CDC-LT & \\
\hline Aedesalbopictus & - & - & 5 & - & 23 & 1 & - & - & 1 & - & 30 \\
\hline Aedescaecus & 19 & 16 & - & - & - & - & - & - & - & - & 35 \\
\hline Aedesniveus & - & - & 3 & 1 & 13 & - & - & - & 32 & - & 49 \\
\hline Anopheles aconitus & 9 & - & 17 & - & - & - & 22 & - & - & - & 48 \\
\hline Anopheles barbirostris & 19 & - & 12 & - & - & - & - & - & - & - & 31 \\
\hline Anopheles barumbrosus & 14 & - & 4 & - & - & - & 9 & - & - & - & 27 \\
\hline Anopheles balabacensis & - & - & - & - & - & - & - & - & 3 & - & 3 \\
\hline Anopheles cracens & 44 & 48 & 18 & 36 & 12 & 8 & 9 & 12 & 12 & 12 & 211 \\
\hline Anopheles crawfordi & 13 & - & - & - & - & - & 7 & - & - & - & 20 \\
\hline Anopheles donaldi & 5 & - & 29 & - & - & - & 15 & - & - & - & 49 \\
\hline Anopheles hodgkini & 19 & - & - & - & - & - & 14 & - & - & - & 33 \\
\hline Anopheles introlatus & - & - & - & - & 38 & 3 & 13 & - & 81 & - & 135 \\
\hline Anopheles karwari & 7 & - & 12 & - & - & - & - & - & - & - & 19 \\
\hline Anopheles kochi & 8 & - & 2 & - & - & - & 5 & - & - & - & 15 \\
\hline Anopheles latens & - & - & - & - & - & - & 1 & - & - & - & 1 \\
\hline Anopheles letifer & - & - & - & - & - & - & - & - & 39 & 2 & 41 \\
\hline Anopheles maculatus & 38 & - & 20 & - & 18 & - & 60 & - & - & - & 136 \\
\hline Anopheles nemophilous & 3 & - & 2 & - & - & - & 2 & - & - & - & 7 \\
\hline $\begin{array}{c}\text { Anopheles } \\
\text { philippinensis }\end{array}$ & 4 & - & 6 & - & - & - & 1 & - & - & - & 11 \\
\hline Anopheles pursati & - & - & - & - & - & - & 15 & - & - & - & 15 \\
\hline Anopheles sinensis & - & - & 1 & - & - & - & 3 & - & - & - & 4 \\
\hline Armigeres bhayungi & - & - & - & - & 14 & - & 34 & - & - & - & 48 \\
\hline Armigeres confuses & 1 & - & 2 & - & - & - & 1 & - & - & - & 4 \\
\hline Armigeres durhami & 9 & 18 & 14 & 9 & - & - & 15 & 12 & - & - & 77 \\
\hline Armigeres moultoni & - & - & - & - & - & - & 10 & - & - & - & 10 \\
\hline Armigeres subalbatus & 17 & - & - & - & - & - & - & - & - & - & 17 \\
\hline Coquillettidia spp. & - & 25 & - & 1 & - & - & - & 13 & 9 & - & 48 \\
\hline Culexgelidus & 23 & - & 54 & - & 46 & - & 19 & - & 60 & - & 202 \\
\hline Culexpseudovishnui & 5 & - & - & - & - & - & - & - & - & - & 5 \\
\hline Culexquinquefasciatus & - & - & 4 & 1 & - & - & - & - & - & - & 5 \\
\hline Culexsitiens & - & - & - & - & 27 & - & 5 & - & - & - & 32 \\
\hline Culexvishnui & 2 & - & - & - & - & - & - & - & - & - & 2 \\
\hline Culexwhitemorei & 1 & - & - & - & - & - & - & - & - & - & 1 \\
\hline Mansoniabonnae & 26 & - & - & 1 & - & - & 18 & - & - & - & 45 \\
\hline TOTAL & 286 & 107 & 205 & 49 & 191 & 12 & 278 & 37 & 237 & 14 & 1416 \\
\hline
\end{tabular}


both methods but not necessarily from the same area as in the case of Coquillettidia spp. and Ma. bonnae. As shown in Table 1, HLC technique collected more mosquito species making $84.5 \%$ of the total mosquito collected as opposed to $\mathrm{CO}_{2}$-baitedCDC-LT technique which collected only $15.5 \%$ of the total mosquito.The number of mosquito collected based on both techniques for all study areas is displayed in Figure 2. Highest number of mosquito was captured in Kg Mela (Kuala Lipis Pahang) (27.8\%), followed by Kg Sokor (Tanah Merah Kelantan) (22.2\%), Lata Cemerong (Dungun Terengganu) (17.8\%), Mersing (Johor) (17.7\%) and Pos Dipang (Kampar Perak) (14.3\%). It was clear that for every study area HLC technique collected higher number of mosquitoes compared to $\mathrm{CO}_{2}$-baited CDC-LT technique. The highest different in number of mosquito captured was in Mersing where HLC technique collected mosquito of almost 17 times more than $\mathrm{CO}_{2}$-baited CDC-LT technique followed by Post Dipang (16 times), Kg Sokor (seven times), Lata Cemerong (four times) and Kg. Mela (almost three times).

An. cracens from all localities was subjected for molecular analysis based on inter-transcribed spacer region 2 (ITS2) of ribosomal DNA [11]-[13] in order to confirm the species. Figure 3 showed that those mosquitoes were confirmed as An. cracens.

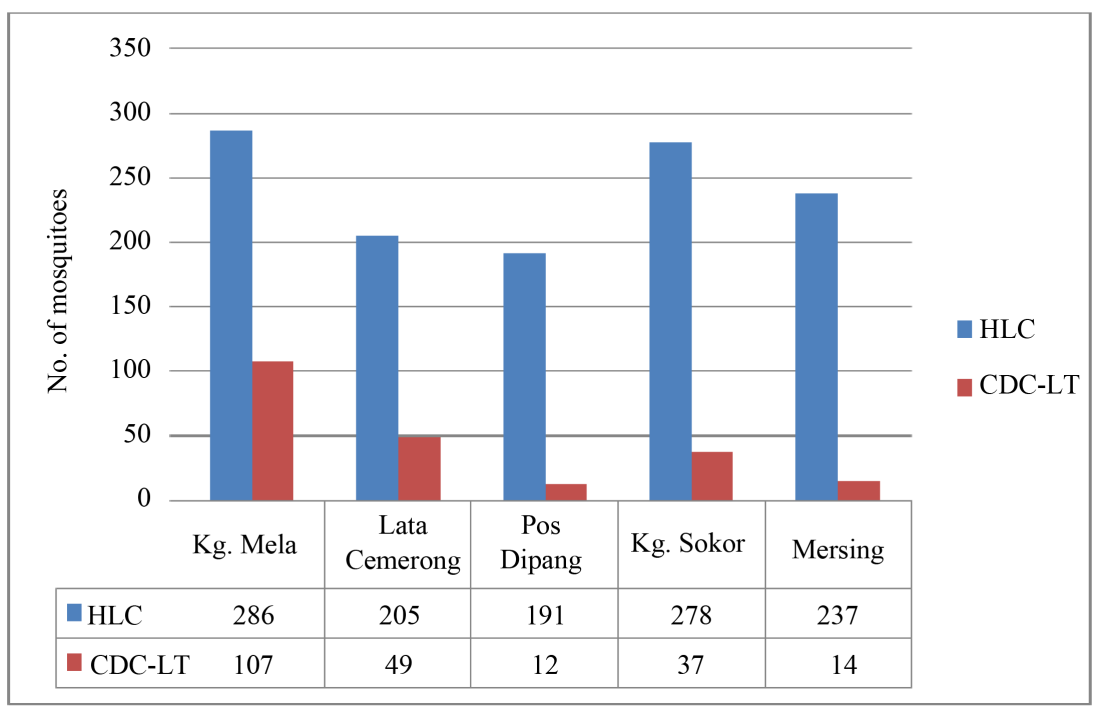

Figure 2. Total number of adult mosquitoes collected per collection method for all areas.

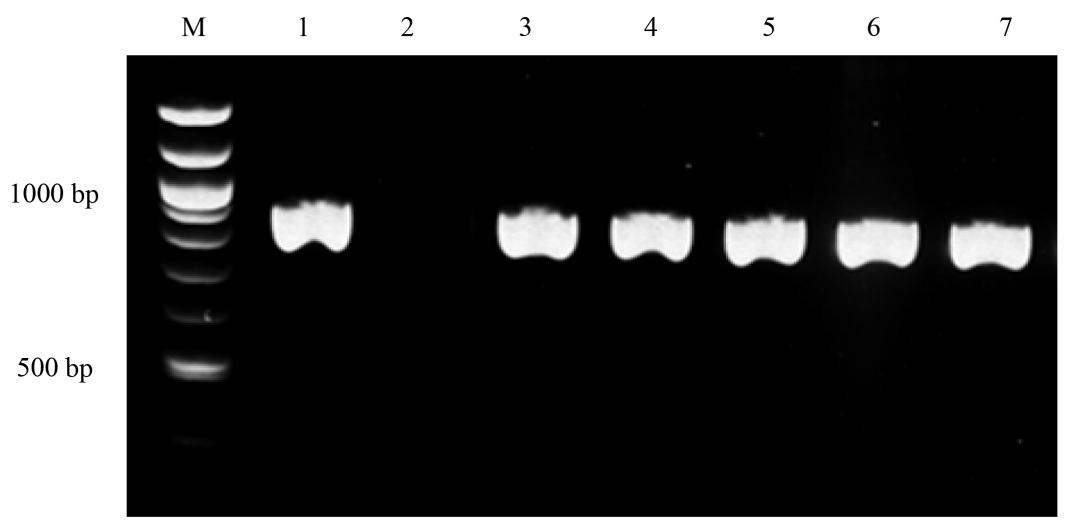

Figure 3. Gel picture showing PCR amplicons generated from the An. cracens. M indicate DNA ladder of 100 bp. Lane 1 (An. cracens Lab strain-positive control), Lane 2 (negative control), Lane 3 (An. cracens Kg. Mela strain), Lane 4 (An. cracens Lata Cemerung strain), Lane 5 (An. cracens Pos Dipang strain), Lane 6 (An. cracens Kg. Sokor strain), Lane 7 (An. cracens Mersing strain). The sizes of amplicons for An. cracens was about 850 bp. 
Figure 4 presents the distribution of An. cracens mosquitoes the primary vector for knowlesi malaria caught in the five areas using the two techniques. An. cracens was found to be the predominant species in Kg. Mela (43.6\%), Lata Cemerung (25.6\%) and Kg Sokor (11.4\%) (Table 1). Interestingly $\mathrm{CO}_{2}$-baited CDC-LT technique was shown capturing more An. cracens than HLC technique in those study areas. $\mathrm{CO}_{2}$-baited CDC-LT technique collected equivalently with HLC technique in Mersing (Johor) but was out numbered in Pos Dipang (Kampar Perak). Anopheles cracens was also the most dominant species captured among the Anopheles species (Table 1). Unlike the other species, An. cracens was captured by both methods at all study sites. This is definitely an interesting finding since this could be the first ever data that indicates An. cracens was caught using $\mathrm{CO}_{2}$-baited CDC-LT technique. In order to generalize the findings, Z proportion Test (for large sample size) and Fisher Exact Test (for small sample size) were conducted and the results are displayed in Table 2. Based on the proportion values for each study areas, $\mathrm{CO}_{2}$-baited CDC-LT technique has the highest proportion (or percentages) ranging from 0.32 to 0.86 (32\% to $86 \%)$ as compared to HLC technique which all values were less than 0.20 (20\%). The Z-Test and Fisher Exact Test showed highly significant p-values which indicate that $\mathrm{CO}_{2}$-baited CDC-LT technique significantly performed efficiently better than HLC technique in capturing knowlesi malaria vector, An. cracens.

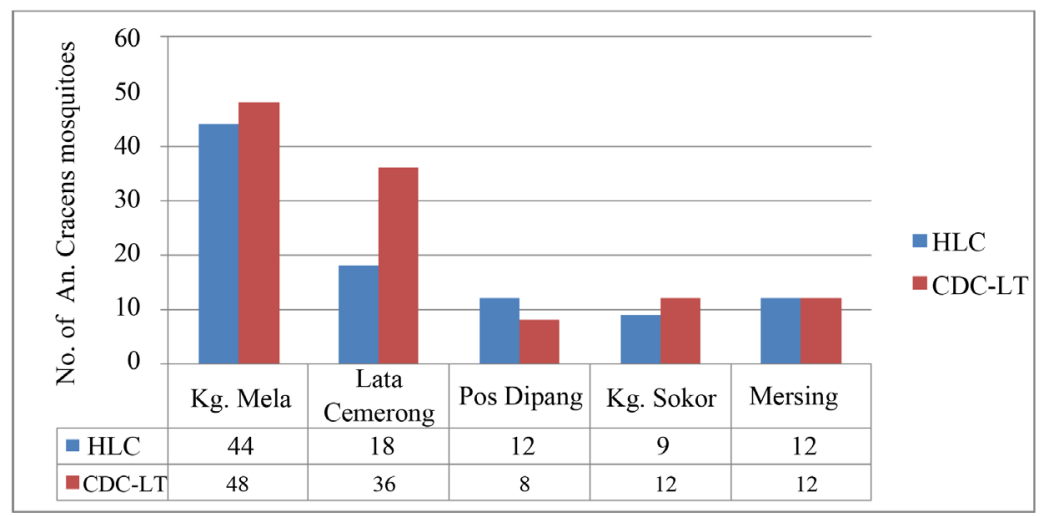

Figure 4. Total number of An. cracens mosquitoes captured using HLC and dry-ice baited CDC-LT techniques for each study area.

Table 2. Proportion test according to five study areas.

\begin{tabular}{|c|c|c|c|c|}
\hline & & HLC & CDC-LT & Proportion test (p-value) \\
\hline \multirow{3}{*}{$\begin{array}{l}\text { 1) Kg. Mela } \\
\text { Kuala Lipis } \\
\text { Pahang }\end{array}$} & An. cracens & 44 & 48 & \multirow{3}{*}{$\begin{array}{c}\text { Z-Test } \\
\mathrm{p}<0.0001^{* * *}\end{array}$} \\
\hline & Total mosquitoes collected & 286 & 107 & \\
\hline & Proportion (Percentages) & 0.15 & 0.45 & \\
\hline \multirow{3}{*}{$\begin{array}{l}\text { 2) Lata Cemerong } \\
\text { Dungun } \\
\text { Terengganu }\end{array}$} & An. cracens & 18 & 36 & \multirow{3}{*}{$\begin{array}{c}\text { Z-Test } \\
\mathrm{p}<0.0001^{* * *}\end{array}$} \\
\hline & Total mosquitoes collected & 205 & 49 & \\
\hline & Proportion & 0.09 & 0.74 & \\
\hline \multirow{3}{*}{$\begin{array}{l}\text { 3) Pos Dipang } \\
\text { Kampar } \\
\text { Perak }\end{array}$} & An. cracens & 12 & 8 & \multirow{3}{*}{$\begin{array}{l}\text { Fischer Exact Test } \\
\mathrm{p}<0.0001^{* * *}\end{array}$} \\
\hline & Total mosquitoes collected & 191 & 12 & \\
\hline & Proportion & 0.06 & 0.67 & \\
\hline \multirow{3}{*}{$\begin{array}{l}\text { 4) Kg. Sokor } \\
\text { Tanah Merah } \\
\text { Kelantan }\end{array}$} & An. cracens & 9 & 12 & \multirow{3}{*}{$\begin{array}{c}\quad \text { Z-Test } \\
\mathrm{p}<0.0001^{* * *}\end{array}$} \\
\hline & Total mosquitoes collected & 278 & 37 & \\
\hline & Proportion & 0.03 & 0.32 & \\
\hline \multirow{3}{*}{ 5) Mersing Johor } & An. cracens & 12 & 12 & \multirow{3}{*}{$\begin{array}{l}\text { Fischer Exact Test } \\
\qquad p<0.0001^{* * *}\end{array}$} \\
\hline & Total mosquitoes collected & 237 & 14 & \\
\hline & Proportion & 0.05 & 0.86 & \\
\hline
\end{tabular}

\footnotetext{
${ }^{* * *}$ Significant at $1 \%$.
} 


\section{Discussion}

This work confirms that the technique used for mosquito collection influences the quantity and the variety of mosquitoes collected. The suitability of mosquito sampling technique may vary alongside geographic and ecological differences, necessitating the calibration of sampling tools for use in different settings. Many studies have evaluated the performance of the $\mathrm{CO}_{2}$-baited CDC-LT technique relative to the HLC technique but it is very difficult to compare the results due to the different methodologies and sampling procedure applied. In this study, the HLC technique consistently collected higher numbers of mosquitoes for most of the mosquito species than the $\mathrm{CO}_{2}$-baited CDC-LT technique, demonstrating that $\mathrm{HLC}$ technique was more efficient than the $\mathrm{CO}_{2}$ baited CDC-LT technique. This finding was in accordance with other studies which suggested that HLC technique is the most appropriate sampling technique for anthropophilic Anopheles mosquitoes [14]-[17]. However, this may vary between species. This study also showed that the predominant species collected were An. cracens, Cx. gelidus, An. maculatus An. introlatus, and Ar. durhami. Although HLC technique was more efficient than the $\mathrm{CO}_{2}$-baited CDC-LT technique in collecting many species of mosquitoes, it was not so in the case of An. cracens. This study clearly showed that the number of $A n$. cracens mosquitoes collected by both techniques was found almost equal. Inthreeout of five study areas (Kg. Mela, Lata Cemerung and $\mathrm{Kg}$ Sokor) $\mathrm{CO}_{2}$-baited CDC-LT technique collected more An. cracens than HCL technique. Statistical analysis performed on all results obtained from all study areas demonstrated that the differences seen between the two techniques was found highly significant indicating that $\mathrm{CO}_{2}$-baited CDC-LT technique performed efficiently better than HLC technique in catching An. cracens mosquitoes.

Most of the Anopheles species were collected almost exclusively by the HCL technique except for An. cracens and An. introlatus. These two species were collected using both techniques. As for An. maculatus, the species incriminated as the vector of malaria in Peninsula Malaysia [1], it was collected mainly using HCL technique in all the study areas except in Mersing (Johor). HLC technique probably was the most efficient technique because this species is highly anthropophilic and less influenced by light attraction [18].

As previously mentioned, the Anopheles species attracted to $\mathrm{CO}_{2}$-baited CDC-LT technique were An. cracens and An. introlatus. Interestingly, An. cracens and An. introlatus had been incriminated as the primary and secondary vectors for knowlesi malaria in peninsula Malaysia respectively [1] [7]. This study therefore provides the evidence that $\mathrm{CO}_{2}$-baited CDC-LT technique was reliable in collecting vectors of $P$. knowlesi especially An. cracens.

The study also showed that, to monitor vector of human malaria, effective sampling technique for host seeking vectors such as HLC technique is necessary. Therefore both HLC and $\mathrm{CO}_{2}$-baited CDC-LT techniques are suitable for sampling vector of knowlesi malaria. These traps however, might not work well in all epidemiological settings and therefore more mosquito behavioural studies should be carried out in order to gain more insight for further development of mosquito sampling and control tools.

In Malaysia, knowlesi malaria transmission appears more strongly associated with land development than water development projects. Changes in land use such as deforestation, increased urbanisation and agriculture development can directly impact mosquito abundance, species biodiversity, biting behaviour and vector competency. Land development increases the number of humans working in an area and can alter environmental conditions that favour mosquitoes [19]. Most of the knowlesi malaria cases reported in peninsula Malaysia was in rural villages either near or border to tropical forest fringes, rubber or oil palm plantations [4]. Commercial plantations tend to offer suitable environmental conditions for Anopheles mosquito breeding. Condition as simple as wheel tracks when contain enough rain water created perfect breeding sites for An. cracens as has been detected by $\mathrm{HLC}$ and $\mathrm{CO}_{2}$-baited CDC-LT technique. Clearly, people living and working in such plantations are most likely to be at risk for knowlesi malaria.

\section{Conclusion}

In order to monitor vector of human malaria, effective sampling techniques for host seeking vectors is necessary. This work confirmed that the technique used in sampling of adult mosquito influences the quantity and the variety of mosquitoes collected. As indicated in this study, HLC technique was shown to be more effective than $\mathrm{CO}_{2}$-baited CDC-LT technique in monitoring transmission of not only malaria but also other mosquito-borne infections. However, as monitoring focused on sampling of the vector of knowlesi malaria An. cracens, $\mathrm{CO}_{2}$-baited CDC-LT technique was found to perform efficiently better than HLC technique. This study con- 
cludes that both techniques are suitable for monitoring An. cracens the vector of knowlesi malaria. In such situation, selection of the sampling technique eventually has to be decided based on technique that presents low operational cost, less logistically demanding, human exposure-free and the aim of the study to be conducted.

\section{Acknowledgements}

The authors are grateful to the Director-General of Health, Malaysia for permission to publish this paper. We especially thanked the staff of Medical Entomology Unit of IMR, without whose diligence and hard work under difficult field conditions this research would not have been accomplished. This study was funded by National Institutes of Health, Ministry of Health Malaysia.

\section{Competing Interests}

The authors declare no competing interests.

\section{Authors' Contributions}

RA, AAAR and ZMN designed and coordinated the study and drafted the manuscript. AAAR, ZI HMO, AMM and ZH carried out the fieldwork and mosquito identifications and analysed the results. WNWMA participated in the design of the study, its coordination and the evaluation. SI carried out statistical analysis. LHL provided advice during the study design and field surveillance implementation. All authors read and approved the final manuscript.

\section{References}

[1] Reid, J.A. (1968) Anopheline Mosquitoes of Malaya and Borneo. Institute for Medical Research Malaysia, Malaysia, $1-520$.

[2] White, N. (2008) Plasmodium knowlesi. The Fifth Human Malaria Parasite. Clinical Infectious Diseases, 46, 172. http://dx.doi.org/10.1086/524889

[3] Cox-Singh, J., Davis, T.M., Lee, K.S., Shamsul, S.S., Matusop, A., Ratnam, S., Rahman, H.A., Conway, D.J. and Singh, B. (2008) Plasmodium knowlesi Malaria in Humans Is Widely Distributed and Potentially Life-Threatening. Clinical Infectious Diseases, 46, 165-171. http://dx.doi.org/10.1086/524888

[4] (2013) Vector Borne Diseases Control Programme (VBCP): Annual Report Ministry of Health. Ministry of Health Malaysia.

[5] Vythilingam, I. (2010) Plasmodium knowlesii in Humans: A Review on the Role of Its Vectors in Malaysia. Tropical Biomedicine, 27, 1-12.

[6] Service, M.W. (1963) An Appraisal of Adult Mosquito Trapping Techniques Used in Nigeria, West Africa. Journal of Tropical Medicine, 27-33.

[7] Oli, K., Jeffery, J. and Vythilingam, I. (2005) A Comparative Study of Adult Mosquito Trapping Using Dry Ice and Yeast Generated Carbon Dioxide. Tropical Biomedicine, 22, 249-251.

[8] Gillies, M.T. (1980) The Role of Carbon Dioxide in Host Finding by Mosquitoes (Diptera: Culicidae): A Review. Bulletin of Entomological Research, 70, 525-532. http://dx.doi.org/10.1017/S0007485300007811

[9] Vythilingam, I., Chiang, G.L. and Chan, S.T. (1992) Evaluation of $\mathrm{CO}_{2}$ and 1-Octen-3-ol as Mosquito Attractant. Southeast Asian Tropical Medicine and Public Health, 23, 328-331.

[10] Sallum, M.A.M., Peyton, E.L., Harrison, B.A. and Wilkerson, R.C. (2005) Revision of the Leucosphyrus Group of Anopheles (Cellia) (Diptera, Culicidae). Revista Brasileira de Entomol, 49, 101-152. http://dx.doi.org/10.1590/S0085-56262005000500001

[11] Sum, J.S., Lee, Y.C., Amirah, A., Kamil, A.B., Jeffery, J., Noraishah, M.A.A., Fong, M.Y. and Lau, Y.L. (2014) Phylogenetic Study of Six Species of Anopheles Mosquitoes in Peninsular Malaysia Based on Inter-Transcribed Spacer Region 2 (ITS2) of Ribosomal DNA. Parasites \& Vectors, 7, 309. http://dx.doi.org/10.1186/1756-3305-7-309

[12] Vythilingam, I., Yvonne, A.L., Venugopalan, B., Romano, N., Cherng, S.L., Meng, L.W., Loke, T.K., Xiang, T.G., Nan, J.Y., Yusoff, W.W.S., Jeffery, J., Ab Ghani, C.T., Zawiah, I., Reuben, S.K.S., Lau, Y.L. and Rohela, M. (2014) Plasmodium knowlesi Malaria an Emerging Public Health Problem in Hulu Selangor, Selangor, Malaysia (2009-2013): Epidemiologic and Entomologic Analysis. Parasites \& Vectors, 7, 436. http://dx.doi.org/10.1186/1756-3305-7-436

[13] Walton, C., Hardley, J.M., Kuvangkadilok, C., Collins, F.H., Harbach, R.E., Baimai, V. and Butlin, R.K. (1999) Iden- 
tification of Five Species of the Anopheles dirus Complex from Thailand, Using Allele-Specific Polymerase Chain Reation. Medical and Veterinary Entomology, 13, 24-32. http://dx.doi.org/10.1046/j.1365-2915.1999.00142.x

[14] Overgaard, H.J., Solve, S., Michael, R., Varnsi, P.R., Simon, A., Abrahan, M. and Micahel, S. (2012) Light Traps Fail to Estimate Reliable Malaria Mosquito Biting Rates on Bioko Island, Equatorial Guinea. Malaria Journal, $11,46$. http://dx.doi.org/10.1186/1475-2875-11-56

[15] Okumu, F., Maya, E.K., Japheth, K., Evan, M., Gerry, F.K. and Sarah, J.M. (2008) Comparative Evaluation of Methods Used for Sampling Malaria Vectors in the Kilombero Valley, South Eastern Tanzania. The Open Tropical Medicine Journal, 1, 51-55. http://dx.doi.org/10.2174/1874315300801010051

[16] Ritchie, S.A. and Kline, D.L. (1995) Comparison of CDC and EVS Light Traps Baited with Carbon Dioxide and Octenol for Trapping Mosquitoes in Brisbane, Queensland. Journal Australia Entomology, 34, 215-218. http://dx.doi.org/10.1111/j.1440-6055.1995.tb01322.x

[17] Mathenge, E.M., Gedion, O., David, O., Lucy, I., Paul, N., Tom, S., Gerry, K. and Bart, K. (2005) Comparative Performance of the Mbita Trap, CDC Light Trap and the Human Landing Catch in the Sampling of Anopheles arabiensis, Anopheles funestus and Culicine Species in a Rice Irrigation in Western Kenya. Malaria Journal, 4, 7. http://dx.doi.org/10.1186/1475-2875-4-7

[18] Ndiath, M.O., Mazenot, C., Gaye, A., Konate, L., Bouganal, C., Faye, O., Sukna, C. and Trape, J.F. (2011) Methods to Collect Anopheles Mosquitoes and Evaluate Malaria Transmission: A Comparative Study in Two Villages in Senegal. Malaria Journal, 10, 270. http://dx.doi.org/10.1186/1475-2875-10-270

[19] Norris, D.A. (2004) Mosquito-Borne Diseases as a Consequence of Land Use Change. Ecohealth, 1, 19-24. http://dx.doi.org/10.1007/s10393-004-0008-7 